\title{
Kryptographie im Wandel
}

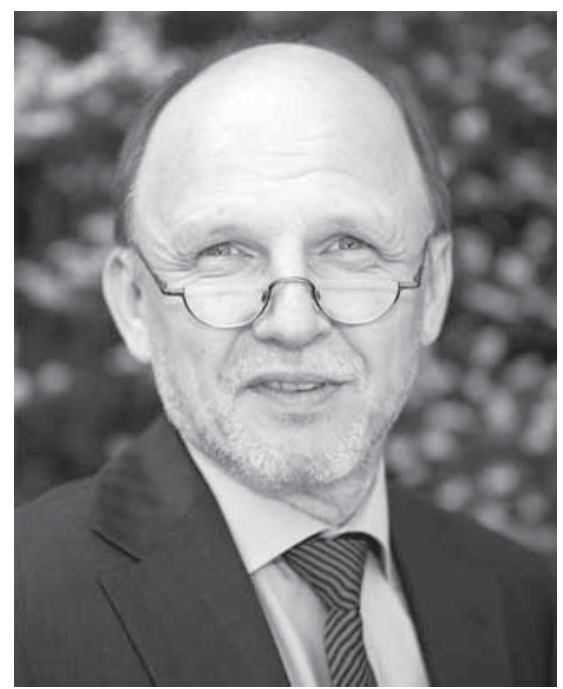

Die Digitalisierung verändert ebenso tiefgreifend wie rasant nahezu alle Bereiche von Wirtschaft, Gesellschaft und täglichem Leben. Während Internet, mobile Kommunikation und digitale Anwendungen längst zu unserem Alltag gehören, erfasst die digitale Transformation derzeit mit enormer Dynamik sämtliche Stufen der industriellen Wertschöpfung, von der Logistik über die Produktion bis hin zur Dienstleistung.

Parallel mit dieser Entwicklung setzen wir immer mehr Kryptographie ein, häufig ohne uns dessen überhaupt bewusst zu sein. Die digitale Infrastruktur ist durchsetzt von Schutzvorkehrungen vielfältiger Art, die überwiegend auf kryptographischen Mechanismen basieren. Deren Wirksamkeit bildet zudem eine der Grundvoraussetzungen der Digitalisierung.

Naturgemäß muss diese Wirksamkeit regelmäßig überprüft werden, nicht zuletzt um mit der Entwicklung der Leistungsfähigkeit von Computern Schritt zu halten. Als eine Alternative zur klassischen Halbleitertechnik haben Quantencomputer in den vergangenen Jahren ein ständig zunehmendes Interesse in Forschung und Industrie erfahren und deren Entwicklung in Folge eine erhebliche Beschleunigung. Da hinreichend leistungsfähige Quantencomputer eine ernst zu nehmende Gefahr für viele international gebräuchliche kryptographische Verfahren darstellen würden, hat das Thema Quantencomputerresistenz deutlich an Aktualität gewonnen.

Diesem spannenden Bereich sind im vorliegenden Heft gleich zwei Beiträge gewidmet. Der Artikel "Quantencomputer und die Zukunft der Kryptographie“ diskutiert die oben angesprochenen Szenarien und skizziert Alternativen zur "klassischen“ Kryptographie, die nach heutigem Wissensstand auch in einem Zeitalter der Quantencomputer Bestand hätten. Mit dieser Diskussion verbindet sich unmittelbar die Frage nach praktischen Auswirkungen und möglichen Ablösestrategien. Der Aufsatz von Kim Nguyen stellt solche Ablöseszenarien vor und setzt sich aus Sicht von Zertifizierungsdiensten kritisch mit Anforderungen an Post-Quantum-Algorithmen und Systeme auseinander.

Während das Zeitalter der Quantencomputer noch in mehr oder weniger weiter Ferne liegt, ist das Zeitalter der Blockchain bereits angebrochen. Auch die Blockchain stellt hohe Anforderungen an die Kryptographie. Die Erwartungen an die BlockchainTechnologie sind extrem hoch - ob sie auch zu erfüllen sind, wird die nähere Zukunft zeigen. Die Abhandlung von Sebastian Stommel leistet einen Beitrag zur Standortbestimmung.

Im privaten Bereich setzt die Nutzung digitaler Anwendungen häufig die Einwilligung in die Verarbeitung und Weitergabe personenbezogener Daten voraus. Gleichzeitig können Nutzer häufig kaum noch nachvollziehen, welche Konsequenzen sich aus ihrer Einwilligung ergeben. Marian Margraf diskutiert in seinem Aufsatz verschiedene Ansätze, die zum Ziel haben, selbstbestimmtes Handeln in der digitalen Welt wieder zu ermöglichen.

Die eIDAS-Verordnung der EU - sozusagen die dritte Generation der Signaturgesetzgebung -zielt auf einfachere Technik, neue Signatur-Varianten und bessere internationale Abstimmung. Klaus Schmeh beschreibt in seinem Beitrag, welche Anforderungen an die Krypto-Technik sich aus diesen Neuerungen ergeben.

Im Artikel "Sicherheit relativ definieren - Das Real/Ideal-Paradigma in der Kryptographie" schließlich wird ein simulationsbasierter Modellierungsansatz vorgestellt, der sich in der modernen Kryptographie als Standardsicherheitsdefinition für kryptographische Protokolle etabliert hat. Das Besondere an diesem Begriff ist, dass Sicherheit durch Vergleich definiert wird und deshalb keine absolute Größe darstellt.

Wir hoffen, das Spektrum der Beiträge in diesem Heft findet Ihr Interesse.

\section{Walter Fumy}

